\title{
Underactuated Gripper that is able to Convert from Precision to Power Grasp by a Variable Transmission Ratio
}

\author{
Stefan A.J. Spanjer ${ }^{1}$, Ravi Balasubramanian ${ }^{2}$, Member, IEEE, Aaron M. Dollar ${ }^{3}$, \\ Member, IEEE, Just L. Herder ${ }^{1}$, Member, IEEE \\ ${ }^{1}$ Laboratory of Mechanical Automation and Mechatronics, Faculty of Engineering Tech- \\ nology, University of Twente, Enschede, Netherlands \\ (e-mail: s.a.j.spanjer@student.utwente.nl,j.l.herder@utwente.nl) \\ ${ }^{2}$ School of Mechanical, Industrial \& Manufacturing Engineering, Oregon State University, \\ Corvallis, OR USA (e-mail: ravi.balasubramanian@oregonstate.edu) \\ ${ }^{3}$ Department of Mechanical Engineering and Materials Science, School of Engineering and \\ Applied Science, Yale University, New Haven, CT USA (e-mail:aaron.dollar@yale.edu).
}

\begin{abstract}
This paper investigates the possibility of converting grasped objects from precision grasps to power grasps using a variable transmission ratio for an underactuated finger. To this effect, a variable radius pulley was designed. A simulation study is presented to analyse grasping behaviour and a potential energy method is used to predict the equilibrium positions of finger and object. With this method stable and unstable equilibrium positions are determined. This is followed by an experiment to verify the theory. This paper is a first step in dextrous manipulation with large movements of objects using underactuated fingers.
\end{abstract}

Keywords underactuated, convert, precision grasp, power grasp, variable transmission ratio.

\section{Introduction}

There exists much research on underactuated robotic hands that show adaptive behavior and therefore these are able to pick up different objects [1-4]. Some underactuated hands are able to pick up objects with an enveloping grasp (power grasp) or with a precision grasp depending on the position of the object with respect to the hand and geometry parameters of the hand $[2,5]$. With the so-called equilibrium point method the type of grasp can be easily predicted [2].

For any grasp type, robustness is an important aspect. Robustness is defined here as the ability to resist external forces [6].

The precision grasp is necessary for a successful grasp when objects are small or hard to pick up. The robustness of a precision grasp is less than the robustness of a power grasp, because the number of contact points is less. In [7] the robustness of the precision grasp is improved by actively varying the transmission ratio of an underactuated finger after an object is grasped. The transmission ratio is 
changed such that the required friction force between finger and object is reduced to zero. This way, the equilibrium margin for unknown disturbances is maximized. Still, for high accelerations of hand and object the power grasp is preferred because of its greater robustness. Ideally a hand should be able to convert from precision grasp to power grasp after an object is grasped, because then both small and large objects can be picked up and the desired robustness can be achieved.

The objective of this paper is to present a way to convert from precision to power grasp. This is achieved by varying the transmission ratio in an underactuated finger after an object is grasped (Fig. 1), which leads to motion of the finger and object. The principle of the variable transmission ratio is the same as in [7], but now this ratio is changed in order to break equilibrium and move the object into the hand: by changing the radius of the proximal pulley of a finger with a tendon-pulley mechanism the equilibrium point can be controllably shifted outwards (Fig. 1). As a consequence the distal link rotates counterclockwise and brings the object into the palm of the hand until equilibrium is reached again. For simplicity, we assume symmetry between the two fingers that hold the object. The object is able to move freely in $\mathrm{y}$-direction while the $\mathrm{x}$-direction is prohibited. The principle of potential energy is used to estimate stable and unstable positions of the object in the hand [8]. Therefore, the theory used in this paper is also applicable to other mechanisms for underactuation, for example four bar mechanisms.

The structure of the paper is as follows. We describe the model of grasp transition and the experiment set-up to validate this model in Sections 2 and 3, respectively. In Section 4 we describe the simulation results and show with the experiment that the finger converts from precision to power grasp. In Sections 5 and 6 the discussion and conclusion follow respectively.

\section{Model of grasp transition}

This section consists of three parts: background about the equilibrium point, a simulation model of the grasping behavior and the potential energy method.

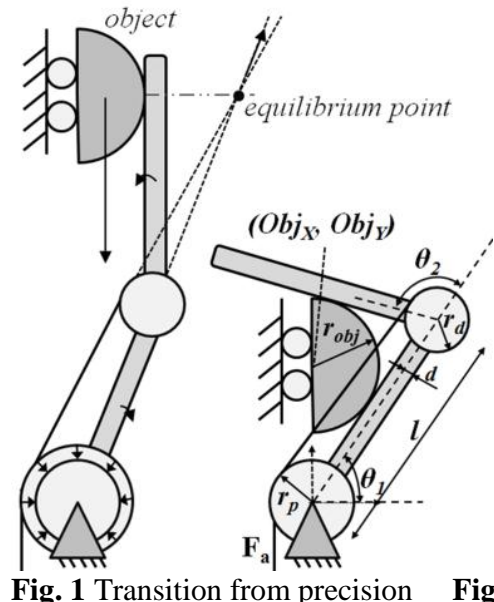

Fig. 1 Transition from precision to power grasp

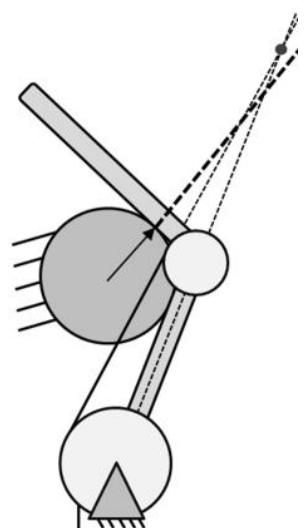

Fig. 2 Unstable mode: force direction leads to caging

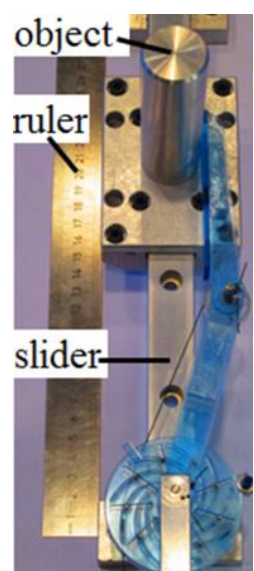

Fig. 3 Experiment set up 
In the model the object is fixed: for different fixed positions the equilibrium of the finger and subsequently the potential energy are estimated. The derivative of the potential energy is equal to the resultant force on the object. This force is zero at points where the derivative is zero. Therefore these points are the positions of the object where the object and finger are in force equilibrium in case the object is not fixed. In the experiment the object is able to move in y-direction (Fig. 1 \& 3) and the force equilibrium positions along this line are validated.

The finger model is based on the model in [7] and consists of a finger and an object at different fixed positions. The finger is tendon driven and consists of two phalanges with length $l=0.1 \mathrm{~m}$ and thickness $2 d=0.015 \mathrm{~m}$, a proximal pulley with adjustable radius $r_{p}$ and a distal pulley with radius $r_{d}=0.01 \mathrm{~m}$ (Fig. 1). It has two torsional springs at the joints with stiffnesses $K_{p}=0.001 \mathrm{Nm}$ and $K_{d}=0.005 \mathrm{Nm}$ and its rest position angles are $\theta_{1, \text { ini }}=25^{\circ}$ and $\theta_{2, \text { ini }}=45^{\circ}$, respectively [9]. The object is a cylinder with radius $r_{o b j}=1.5 \mathrm{~cm}$ and its position is given by $\boldsymbol{O} \boldsymbol{b j}=\left(x_{o b j}, y_{o b j}\right)^{T}$.

\subsection{Equilibrium point}

In Fig. 1 the equilibrium point is drawn. This is the intersection of the tendon line and the proximal phalanx line. The sliding direction and rotation of the distal phalanx on the object can be easily verified with use of the equilibrium point. If the contact force produces a clockwise moment around the equilibrium point, then the distal phalanx will rotate clockwise and vice versa. There is equilibrium if the contact force points at the equilibrium point. In this paper the projection of the equilibrium point on the distal phalanx is called the projected equilibrium point.

\subsection{Grasping process}

The finger contacts the object initially with the proximal or distal phalanx. Subsequently the finger slides along the object until equilibrium is reached or contact is lost. In this section the grasping process is described. Friction is not included in the model.

\subsubsection{Identifying contact mode}

Proximal contact

The following equations are used in case the proximal link makes first contact with the object:

$a_{1}\left\{\begin{array}{c}\cos \left(\theta_{1, i n i}+\delta \theta_{1}\right) \\ \sin \left(\theta_{1, \text { ini }}+\delta \theta_{1}\right)\end{array}\right\}-\left(r_{o b j}+d\right)\left\{\begin{array}{c}\cos \left(\theta_{1, i n i}+\delta \theta_{1}-\pi / 2\right) \\ \sin \left(\theta_{1, \text { ini }}+\delta \theta_{1}-\pi / 2\right)\end{array}\right\}-\left\{\begin{array}{l}x_{o b j} \\ y_{o b j}\end{array}\right\}=\mathbf{0}$

where $\delta \theta_{1}$ is the angle between object and proximal link and $a_{1}$ is the contact position on the proximal link. The required actuation force to make contact is calculated as follows:

$$
F_{a}=\frac{K_{p} \delta \theta_{1}}{r_{p}}
$$

After the proximal phalanx has made contact with the object, the additional actuation force is also calculated with (2) where $\delta \theta_{1}$ and $r_{p}$ are replaced by the angle between object and distal phalanx and $r_{d}$ respectively. This also results in a contact force exerted by the object on the proximal link $F_{c, p}$. The force exerted by the ob- 
ject on the distal link $F_{c, d}$ is equal to zero, because the distal phalanx just touches the object. Now the finger has contact with the object at two points. The equations used for a further increase of the actuation force are described in Section 2.2.3.

\section{Distal contact}

If the distal link makes contact first with the object instead of the proximal link, then the following equations are used:

$$
\begin{gathered}
\delta \theta_{1}-\delta \theta_{2} \frac{K_{d}}{K_{p}} \frac{r_{p}}{r_{d}}=0 \\
l\left\{\begin{array}{l}
\cos \left(\theta_{1, \text { ini }}+\delta \theta_{1}\right) \\
\sin \left(\theta_{1, \text { ini }}+\delta \theta_{1}\right)
\end{array}\right\}+a_{2}\left\{\begin{array}{l}
\cos \left(\theta_{12, i n i}+\delta \theta_{12}\right) \\
\sin \left(\theta_{12, i n i}+\delta \theta_{12}\right)
\end{array}\right\}- \\
\left(r_{o b j}+d\right)\left\{\begin{array}{l}
\cos \left(\theta_{12, i n i}+\delta \theta_{12}-\pi / 2\right) \\
\sin \left(\theta_{12, i n i}+\delta \theta_{12}-\pi / 2\right)
\end{array}\right\}-\boldsymbol{O b j} \mathbf{j}=\mathbf{0}
\end{gathered}
$$

where $\theta_{12, \text { ini }}=\theta_{1, \text { ini }}+\theta_{2, \text { ini }}$ and $\delta \theta_{12}=\delta \theta_{1}+\delta \theta_{2}$ and $a_{2}$ is the contact location on the distal link. The required actuation force to make contact is calculated with (2). The equations used for a further increase of the actuations force are described in Section 2.2.3.

\subsubsection{Contact kinematics}

The contact constraints are derived according to [10]. From analysis it appears that for double contact (proximal and distal) the constraints result in $\Delta a_{2}=\Delta \theta_{1}=$ $\Delta \theta_{2}=0$. The contact constraints for single contact are as follows:

$$
\begin{gathered}
-\Delta a_{2}-\frac{\Delta \theta_{1}\left(r_{o b j}+d\right) l \cos \left(\theta_{2}\right)}{a_{2}}-\Delta \theta_{1} l \sin \left(\theta_{2}\right)=0 \\
-\Delta \theta_{2}-\frac{\Delta \theta_{1} l \cos \left(\theta_{2}\right)}{a_{2}}-\Delta \theta_{1}=0
\end{gathered}
$$

$d$ is the thickness of the finger and $\Delta a$ is the difference in contact location on the distal link.

\subsubsection{Static equilibrium during contact}

\section{Distal contact only}

The quasi-static method is equivalent to the quasi-static procedure in [7]. The following equations are used:

$$
-\boldsymbol{J}_{c}^{T} \Delta \boldsymbol{F}_{c}-\boldsymbol{K} \boldsymbol{\Delta} \boldsymbol{\theta}+\boldsymbol{J}_{a}^{T} \Delta F_{a}=0
$$

$\boldsymbol{K}=\operatorname{diag}\left(K_{p}, K_{d}\right)$ is the stiffness matrix and the matrix $\boldsymbol{J}_{\boldsymbol{a}}=\left(r_{p}, r_{d}\right)$ maps an increase in the actuation force $\Delta F_{a}$ to joint torques. The force exerted by the object on the finger increases with $\Delta \boldsymbol{F}_{\boldsymbol{c}}=\left(\Delta F_{c, p}, \Delta F_{c, d}\right)^{T}$. The matrix $\boldsymbol{J}_{\boldsymbol{c}}$ transfers the contact forces to joint torques [2]:

$$
J_{c}=\left[\begin{array}{cc}
a_{1} & 0 \\
a_{2}+l \cos \theta_{2} & a_{2}
\end{array}\right]
$$

$\boldsymbol{\Delta} \boldsymbol{\theta}=\left(\Delta \theta_{1}, \Delta \theta_{2}\right)^{T}$ is the vector containing the difference in angles as a result of an increment of the actuation force $\Delta F_{a}$. Because there is only contact with the distal phalanx, the increment of the force on the proximal link $\Delta F_{c, p}$ is equal to zero (the 
total force $F_{c, p}$ is also equal to zero). Together with (7) the contact constraint equations (5) and (6) are used in the quasi-static approach. After every iteration $a_{2}, \theta_{1}$, $\theta_{2}$ and $\boldsymbol{J}_{\boldsymbol{c}}$ are updated. The iteration procedure is stopped when the increments $\Delta a_{2}, \Delta \theta_{1}$ and $\Delta \theta_{2}$ are very small which means that the finger has reached an equilibrium state or the procedure is stopped when contact is lost $\left(a_{2}>l\right)$.

The equilibrium point can be used to investigate how the finger will slide along the object. If $F_{c, d}$ produces a clockwise moment about the equilibrium point, then the distal phalanx rotates clockwise and slides until $F_{c, d}$ points in the direction of the equilibrium point or until contact is lost. This is also true in case $F_{c, d}$ produces a counterclockwise moment, except that the finger rotates counterclockwise.

\section{Two point contact (proximal and distal)}

A quasi-static method is used to calculate the contact forces as a consequence of a further increase of the actuation force. Eq. (7) is used again, but now without the product $\boldsymbol{K} \boldsymbol{\Delta} \boldsymbol{\theta}$. The unknowns are $\Delta F_{c, p}$ and $\Delta F_{c, d}$. After every iteration the total forces $F_{c, p}$ and $F_{c, d}$ are updated. There are 2 options: $F_{c, p}$ increases (stable) or decreases (unstable). The finger does not move as long as $F_{c, p}$ is positive. The quasi-static procedure is stopped when $F_{c, p}$ is zero or $F_{c, p}$ and $F_{c, d}$ are sufficiently large. When $F_{c, p}$ is zero and $F_{a}$ is increased, the proximal phalanx loses contact with the object and the finger shows caging behavior [11]: the proximal phalanx rotates clockwise and the distal phalanx rotates counterclockwise. Single contact by the distal link is already modeled in section 2.2.3: eq. (7) is used in combination with the contact constraints in (5) and (6).

The equilibrium point can also be used to see if caging behavior will occur [2]. If $F_{c, d}$ produces a clockwise moment around the equilibrium point, then the finger is in a stable mode (Fig. 2). Caging behavior occurs when $F_{c, d}$ produces a counterclockwise moment around the equilibrium point.

\subsection{Potential Energy}

The gradient of the potential energy is equal to the resultant force on the object. The partial derivative of the potential energy with respect to degree of freedom $y$ is the force in $y$-direction. With the potential energy method the potential energy as a function of the object position can be obtained. For every object position the model is simulated to determine the equilibrium state of the finger. Every time the potential energy of this equilibrium is calculated:

$$
E_{P}=F_{a} \Delta h+\frac{1}{2} K_{p}\left(\theta_{1}-\theta_{1, i n i}\right)^{2}+\frac{1}{2} K_{d}\left(\theta_{2}-\theta_{2, i n i}\right)^{2}
$$

The total actuation force $F_{a}$ is considered constant for different positions. $\theta_{1, \text { in }}$ and $\theta_{2, i n i}$ are the rest position angles at the proximal and distal joint respectively; at this position the torsion springs perform no work. $\Delta h$ is the change in tendon length:

$$
\Delta h=r_{p}\left(\theta_{1}-\theta_{1, \text { ini }}\right)+r_{d}\left(\theta_{2}-\theta_{2, \text { ini }}\right)
$$

For every object position the potential energy is calculated. The potential energy can be displayed as a function of the y-position of the object. Different energy curves can be obtained for different radii of the proximal pulley. 


\section{Experiment set-up}

An experiment was set up to verify that the finger and object can convert from precision to power grasp by changing the transmission ratio. The experiment was also used to validate the end position of the object which is equal to the position of the minimum of the potential energy curve. The parameters are the same as in the model, only the joint stiffnesses are different: $K_{p}=0.015 \mathrm{Nm}$ and $K_{d}=$ $0.048 \mathrm{Nm}$.

The setup of the experiment is shown in Fig. 3. The same acrylic finger is used as in [7] where music-wire springs are mounted at the proximal and distal joint . The constant actuation force is supplied by a $1 \mathrm{~kg}$ weight.

The radius of the proximal pulley is adjustable by rotating a top and bottom plate with respect to one another (Fig. 4). Shafts around which the tendon is routed (i.e. the effective pulley) are located between the straight slots in the bottom plate and the curved slots in the top plate. As the plates rotate with respect to one another, the shafts move radially, changing the effective pulley radius. On the shafts small pulleys are mounted. The logarithmic spiral slots are designed to have a relatively large angle with the radial slots such that friction for adjustment of the pins is low but high enough to make the adjustment system nonbackdrivable. The whole system is connected to the proximal shaft with bearings, so it can rotate freely.

The start position of the finger in the experiment is the precision mode: the finger touches the object by the distal link and is in an equilibrium state (Fig. 3). The y-position of the object depends on the radius of the proximal pulley, so that the object is located at the level of the equilibrium point. In the next step the radius is lowered until the object starts to move. The end position of the object will be compared with the expected minimum of the energy curve of that radius.

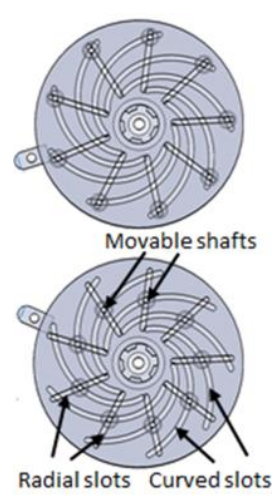

Fig. 4 Variable radius

\section{Results}

\subsection{Simulation results}

Fig. 5 shows potential energy curves for different radii of the proximal pulley. In case a curve has no extreme, it was not possible to establish contact. It is clear that the curve with $r_{p}=0.03 \mathrm{~m}$ has a minimum and a maximum. The stable minimum is the position of the object when finger and object are in power grasp mode. Small disturbances will lead to other positions, but the object will return at the point with minimum energy. The same curve shows also an unstable maximum at $y=0.14 \mathrm{~m}$. When only the distal phalanx has contact with the object, equilibrium will be reached when the contact force does not have a component in y-direction. When the radius is changed, the derivative at $y=0.14 m$ deviates from zero which means a force develops which tends to move the object. Decrease of the radius results in a more distal location of the equilibrium point. As a result, the distal phalanx rotates into a power grasp. 


\subsection{Experimental results}

From the experiment it is clear that the object moves to the proximal phalanx [12]. Fig. 6 shows different trials of the experiment. The start- and end positions of the object together with the start- and end radii of the proximal pulley are depicted. The expected start- and end positions from the simulation results are also shown.

\section{Discussion}

The potential energy method is a simple method to estimate stable and unstable equilibrium positions. Simulation results predict equilibrium positions well.

In Fig. 6 the differences between simulation and experiment in precision grasp (average of $19 \mathrm{~mm}$ ) are larger than the differences in power grasp (average of 3.4 $\mathrm{mm}$ ) for a finger with link length $100 \mathrm{~mm}$ and an object with radius $15 \mathrm{~mm}$. This is probably due to friction, which is not modeled and has more influence in a precision grasp.

In finger object interaction without friction, the equilibrium of the precision grasp is lost for an infinitesimal small proximal radius change. When friction is included, the equilibrium point has to be shifted outside the friction cone. The required radius change to convert from precision to power grasp increases for a larger coefficient of friction between finger and object.

The principle of conversion between precision and power grasp is analyzed and experimented with one finger. Because of symmetry, we assume the theory is also valid for a gripper with two identical fingers. Then the object is also able to move in $\mathrm{x}$-direction and this could lead to asymmetric grasps. The result of radii change for an asymmetric precision grasp is that the fingers convert at different times, because the equilibrium points and force directions per finger are different. When the first finger converts, the object moves and the other finger(s) follow.

The underactuated finger needs an extra actuator for the radius change of the pulley. An advantage of this with respect to fully actuated hands is that the finger shows adaptive behavior and no sensors are needed in the fingers to measure the applied forces.

The potential energy method and the theory of transition between precision and power grasp is demonstrated for a finger with a pulley-tendon mechanism. These methods are also applicable for other types of underactuated fingers, e.g. the equilibrium point of a four bar mechanism could be shifted through a slider

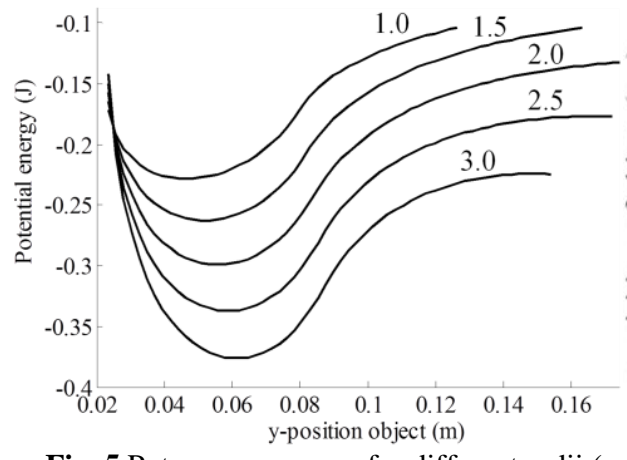

Fig. 5 Pot. energy curves for different radii $(\mathrm{cm})$

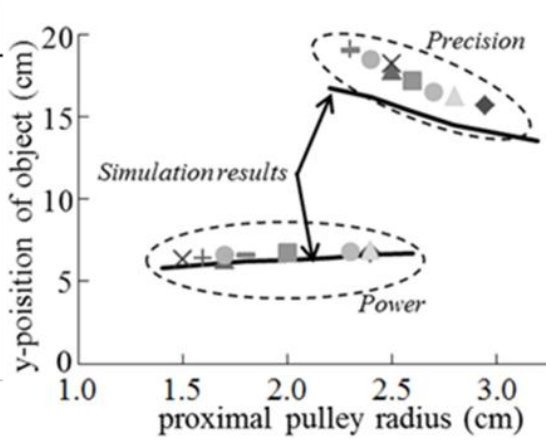

Fig. 6 Experimental results 
that has replaced one of the bars.

From Fig. 5 it appears that the minimum of the potential energy curve with a radius of $10 \mathrm{~mm}$ is at the same level as the maximum of the curve with a radius of $30 \mathrm{~mm}$. A higher radius results in an even lower maximum. Other actuation forces also result in different curves. In this paper we investigated the method to convert from precision to power, but maybe it is also possible to convert from power to precision. It would be very interesting to investigate this dexterous manipulation and to see if it is possible to reposition the object at every position in the hand.

The radii of the pulleys in Hirose's two-fingered soft gripper with 10 phalanges per finger are designed such that objects can be grasped with uniform pressure along the whole finger [1]. If we replace every pulley by a pulley with variable radius in the soft gripper, it may be possible to pick an object with the gripper and move it all the way back to the proximal link by controlling the different radii.

\section{Conclusion}

In this paper we showed an underactuated finger that is able to convert from precision to power grasp by a change of the transmission ratio. With the potential energy method the start position (precision grasp) and the end position (power grasp) of the object are predicted and these are verified with an experiment. The differences between simulations and experiment are about $19 \mathrm{~mm}$ for precision grasps and $3.4 \mathrm{~mm}$ for power grasps for a finger with link length $100 \mathrm{~mm}$ and an object with radius $15 \mathrm{~mm}$.

\section{REFERENCES}

1. S. Hirose and Y. Umetani. The development of soft gripper for the versatile robot hand. Mechanism and Machine Theory, 13:351-359, 1978.

2. L. Birglen, T. Laliberté, and C. Gosselin.Underactuated Robotic Hands. Springer, 2008.

3. A. M. Dollar and R. D. Howe. The Highly Adaptive SDM Hand: Design and Performance Evaluation, Int. Journal of Robotics Research, vol. 29(5), pp. 585-597, 2010.

4. C. Meijneke, G. A. Kragten, and M. Wisse, "Design and performance assessment of an underactuated hand for industrial applications," IFToMM/ASME International Workshop on Underactuated Grasping. pp. 9-15, 2011

5. Delft Hand 3, http://compliantmechanisms.3me.tudelft.nl/mw/index.php/Video DH3

6. Kragten, G. A., \& Herder, J. L. "The ability of underactuated hands to grasp and hold objects." Mechanism and Machine Theory, 45, 408-425, 2010.

7. S. A. J. Spanjer, R. Balasubramanian, J. L. Herder and A.M. Dollar. Improved Grasp Robustness through Variable Transmission Ratios in Underactuated Fingers. IEEE International Conference on Robotics and Automation (ICRA), submitted.

8. Kragten G.A. (2011), "Underactuated Hands: Fundamentals, Performance Analysis and Design", PhD dissertation, Delft University of Technology.

9. Dollar, A. M., \& Howe, R. D. (2005). Towards grasping in unstructured environments: grasper compliance and configuration optimization. Advanced Robotics, 19(5), 523-543.

10.D. J. Montana, "The Kinematics of Contact and Grasp," The International Journal of Robotics Research, vol. 7, no. 3, pp. 17-32, Jun. 1988.

11.R. Balasubramanian, J.T. Belter, \& A.M. Dollar. "External Disturbances and Coupling Mechanisms in Underactuated Hands", International Design Engineering Technical Conferences \& Computers and Information in Engineering Conference. 2010.

12. The VTR Finger, 'youtube video link'. 